\title{
ORIGINAL ARTICLE Relevance of non-nutritional calories in mechanically ventilated critically ill patients
}

\author{
E Bousie $^{1}$, D van Blokland ${ }^{1}$, HJW Lammers ${ }^{2}$ and ARH van Zanten ${ }^{1}$
}

BACKGROUND/OBJECTIVES: Overfeeding in critically ill patients is associated with many complications. Propofol, dextrose infusion and citrate dialysis provide non-nutritional calories (NNCs), potentially causing overfeeding. The relevance of NNCs for total caloric intake has not been systematically studied.

SUBJECTS/METHODS: We retrospectively studied adult mechanically ventilated intensive care unit (ICU) patients receiving enteral nutrition with or without supplemental parenteral nutrition. Primary outcome was the proportion of NNCs (from dextrose, propofol and trisodium citrate) to the total energy intake during the first 7 days after ICU admission. In addition, NNC intake groups were compared.

RESULTS: In total, we identified 146 patients: 142 patients with NNC median value of 580 kcal (interquartile range 310-1043 kcal) over 7 days and 4 patients without NNC intake. The mean proportion of NNCs was larger during the first days after ICU admission (30.7-36.1\%), because of the start-up phase of the nutrition. In the 'propofol' group and the 'dextrose' group this proportion levelled off at $6 \%$ on day 4 . A more stable proportion of $18 \%$ was found during the first 7 days of ICU admission in the 'citrate' group.

CONCLUSIONS: The mean proportion of NNCs in patients who receive dextrose and/or propofol is low (6\%); however, in individual patients it may comprise one-third of the total daily calories. Patients receiving trisodium citrate have higher mean non-nutritional intakes (18\%). As NNC intake can be marked in individual patients, close monitoring is warranted when administering high-dose propofol or trisodium citrate anticoagulation to prevent overfeeding.

European Journal of Clinical Nutrition (2016) 70, 1443-1450; doi:10.1038/ejcn.2016.167; published online 14 September 2016

\section{INTRODUCTION}

Overfeeding in critically ill patients is associated with many complications, such as hyperglycaemia due to increased insulin resistance, hepatic steatosis, infectious morbidity and even mortality. Furthermore, overfed patients have an increased intensive care unit length of stay (ICU LOS) at least partly because of longer duration of mechanical ventilation. ${ }^{1,2}$ Although heavily debated, a recent study showed that withholding nutrition preserved autophagy in the first week of ICU admission; however, others believe that this is poorly substantiated and should not interfere with the delivery of early enteral nutrition during critical illness. ${ }^{3}$

Propofol, dextrose infusion and trisodium citrate anticoagulation during renal replacement therapy can be considered as the major sources of NNCs. When these calories are not taken into account, patients may be at risk of overfeeding.

Dextrose contains $4 \mathrm{kcal} / \mathrm{g}$. Therefore, a $5 \%$ dextrose infusion of $1 \mathrm{~L}(5 \mathrm{~g}$ per $100 \mathrm{ml})$ provides $4 \times 5 \times 10=200 \mathrm{kcal}^{4}{ }^{4}$ The currently available preparation of propofol contains $1-2 \%$ propofol, $10 \%$ soya bean oil, $1.2 \%$ purified egg phospholipid as an emulsifier, $2.25 \%$ glycerol as a tonicity-adjusting agent and sodium hydroxide to adjust the $\mathrm{pH}$. Propofol contains $1.1 \mathrm{kcal} / \mathrm{ml} .^{5}$ Therefore, at an infusion rate of $20 \mathrm{ml} / \mathrm{h}$ over $24 \mathrm{~h}$, the caloric value of the propofol infusion is $20 \times 1.1 \times 24=528 \mathrm{kcal}$.

Trisodium citrate $\left(\mathrm{Na}_{3} \mathrm{C}_{6} \mathrm{H}_{5} \mathrm{O}_{7}\right)$, frequently used as a regional anticoagulant during continuous renal replacement therapy
(CRRT), is another source of energy. The quantity of energy depends on a number of factors (i.e., the concentration used). We use trisodium citrate containing $0.59 \mathrm{kcal} / \mathrm{mmol}(=3 \mathrm{kcal} / \mathrm{g})$. The actual caloric load due to citrate dialysis is more difficult to determine as it depends on a number of factors such as the concentration of trisodium citrate used, the infusion rate, the blood flow in the filter, the filtration fraction into the ultrafiltrate over time (Sieving coefficient), ${ }^{6}$ the ultrafiltrate production per hour and the filter characteristics. Citrate is rapidly metabolised in the citric acid cycle, particularly in the liver, muscles and renal cortex. ${ }^{7}$ We calculated citrate exposition based on a Sieving coefficient of 0.22 , and the actual citrate administration rate, ultrafiltrate production and blood flow in individual patients on an hourly basis.

We designed a study to evaluate the relevance of the actual intake of NNCs among adult critically ill mechanically ventilated patients during the first week after ICU admission, for this study limited to the calories from dextrose infusions, propofol administration and trisodium citrate during CRRT (continuous venovenous haemofiltration in our ICU), among adult critically ill mechanically ventilated patients during the first week after ICU admission. We addressed the daily contributions of NNCs to the total caloric intake, the variability and the importance of the three NNC sources to identify patients at risk of overfeeding because of NNC administration when combined with artificial nutrition.

\footnotetext{
'Department of Intensive Care Medicine, Care Division, Gelderse Vallei Hospital, Ede, The Netherlands and ${ }^{2}$ Department of Clinical Pharmacy, Gelderse Vallei Hospital, Ede, The Netherlands. Correspondence: Dr ARH van Zanten, Department of Intensive Care Medicine, Care Division, Gelderse Vallei Hospital, Willy Brandtlaan 10 , Ede 6716 RP, The Netherlands. 


\section{MATERIALS AND METHODS}

Study design

We performed a retrospective analysis in a mixed medical-surgical ICU in a university-affiliated general teaching hospital (Gelderse Vallei Hospital, Ede, The Netherlands).

\section{Patient population}

The patient database of a retrospective pre-post study evaluating the impact of an electronic nutrition protocol on the adequacy of nutrition delivery in our ICU was used. ${ }^{8}$ Therefore, inclusion criteria were similar: adult mechanically ventilated critically ill patients $(\geqslant 18$ years and ventilated for at least $72 \mathrm{~h}$ ), admitted to the ICU for at least $72 \mathrm{~h}$, receiving enteral and/or parenteral feeding. Exclusion criteria were readmission during the same hospital stay, oral feeding or oral feeding commenced during the first 7 days of ICU admission. In addition, patients discharged to another hospital were excluded.

\section{Data extraction}

Baseline characteristics were listed: age at admission, gender, primary admission diagnosis, baseline Acute Physiology and Chronic Health Evaluation (APACHE II) and Sequential Organ Failure Assessment (SOFA) scores, several baseline blood tests, sepsis diagnosis, admission type (medical, elective and non-elective surgery), comorbidities, Nutrition Risk in Critically ill (NUTRIC) score ${ }^{9}$ and percentage of patients who received NNCs (dextrose infusion (5\% or more), propofol and renal replacement therapy with trisodium citrate).

\section{Outcomes}

Primary outcome was the proportion of NNCs to the total caloric intake during the first 7 days after ICU admission. In case patients were discharged earlier, we only used the data available during ICU admission. NNCs were identified as calories from dextrose, propofol and trisodium citrate. To facilitate clinical identification of patients at risk in a second analysis, we assigned patients to specific subgroups: patients who received dextrose infusion during at least 1 day in the first 7 days of ICU admission ('dextrose' group). Similarly, propofol and citrate patients were grouped ('propofol' group and 'citrate' group, respectively). As a consequence, patients may have been enrolled in more than one group. Furthermore, these three subgroups were compared with the respective patients without administration of dextrose, propofol or trisodium citrate NNCs. To test the impact of the protocol change, we tested whether the total intake of both nutritional and NNCs was nonsignificant comparing both periods to be able to combine all results.

\section{Prediction model}

To be able to identify patients with high cumulative NNC intake during the first week, we performed univariate analyses including the following covariates into the model: age, gender, length and weight at admission, body mass index, days between hospital admission and ICU admission, diagnosis group (cardiovascular, gastrointestinal, haematological, metabolism, neurological, renal or respiratory), APACHE II score, SOFA score, sepsis, admission type (medical, elective surgery, urgent surgery), number of comorbidities, Malnutrition Universal Screening Tool (MUST) score, NUTRIC score and blood tests on the first day of ICU admission (haemoglobin, leucocytes, thrombocytes, creatinine, sodium, potassium, phosphate, albumin, magnesium, bilirubin and glucose). For this analysis, we only used patients with an ICU LOS of 7 days or more. Subsequently, we inserted only univariate covariates with $P$ levels $<0.10$ into a multivariate analysis to predict NNC intake over the first week. The results were used to design an NNC prediction model of baseline characteristics. We decided to use an energy intake from NNCs of at least $5 \mathrm{kcal} / \mathrm{kg}$ during the first 7 days of ICU admission as clinically relevant.

\section{Ethics approval}

The institutional review board of Gelderse Vallei Hospital approved the study and waived informed consent for reasons of the retrospective design and anonymisation of patient identifiers before analysis.

\section{Statistical analysis}

Descriptive data were reported as means and s.d. or median and interquartile range (IQR) in case of skewed distributions, frequencies and percentages or ranges (minimum-maximum). Continuous variables were analysed using independent-sample $T$-test. Univariate and multivariate models were tested using stepwise logistic regression tests. All statistical analysis were made using IBM SPSS Statistics for Windows, version 22.0 (released 2013; IBM Corporation, Armonk, NY, USA). The a priori level of significance was set at $P<0.05$.

\section{RESULTS}

\section{Baseline characteristics}

The patient database of a previous study with a pre-post design was used. No significant differences were observed between the two groups (before and after protocol implementation) in NNCs as percentage of the total caloric intake during the first week of ICU admission: mean of $9.3 \%$ (s.d. \pm 8.2 ) before implementation vs $10.4 \%$ (s.d. \pm 12.6$)$ after implementation $(P=0.52)$. There were also no significant differences between the specific subgroups (citrate and non-citrate, dextrose and non-dextrose and propofol and non-propofol) (see Table 1). Therefore, we have taken the two groups together into one group.

A total of 146 patients were included in the study: 90 men $(61.6 \%)$ and 56 women, with a mean age of 65.9 years (s.d. \pm 14.3 ). Of these patients, 39\% were admitted to the ICU for cardiovascular reasons, followed by respiratory causes (29.5\%) and gastrointestinal diagnoses (21.9\%). The mean baseline APACHE II score was 21.5 (s.d. \pm 7.5 ), and the median baseline SOFA score was 3.0 (IQR 2.0-5.0). Of all patients, $42.5 \%$ were diagnosed with sepsis.

Table 1. Proportion of non-nutritional calories of the caloric target during the first 7 days of ICU admission ${ }^{a}$

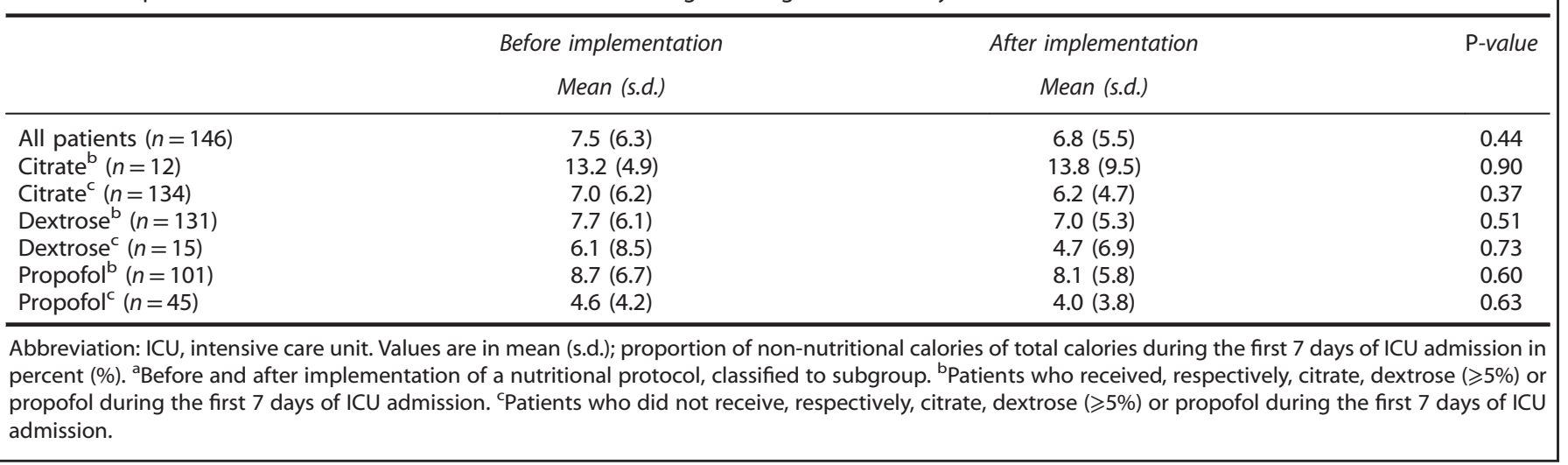


Table 2. Patient characteristics

\begin{tabular}{|c|c|}
\hline Age (years), mean (s.d.) & $65.9(14.3)$ \\
\hline Gender, male, $N(\%)$ & $90(61.6)$ \\
\hline BMI $\left(\mathrm{kg} / \mathrm{m}^{2}\right)$, mean (s.d.) & $26.9(5.9)$ \\
\hline \multicolumn{2}{|l|}{ Primary admission diagnosis } \\
\hline Cardiovascular, N (\%) & $57(39.0)$ \\
\hline Gastrointestinal, $N(\%)$ & $32(21.9)$ \\
\hline Metabolic, $N(\%)$ & $1(0.7)$ \\
\hline Neurological, $N(\%)$ & $12(8.2)$ \\
\hline Renal, $N(\%)$ & $1(0.7)$ \\
\hline Respiratory, $N(\%)$ & $43(29.5)$ \\
\hline Baseline APACHE II score, mean (s.d.) & $21.5(7.5)$ \\
\hline Baseline SOFA score, median (Q1-Q3) & $3.0(2.0-5.0)$ \\
\hline Sepsis, yes, $N(\%)$ & $62(42.5)$ \\
\hline \multicolumn{2}{|l|}{ Admission type } \\
\hline Medical, N (\%) & $91(62.3)$ \\
\hline Elective surgery, $N(\%)$ & $23(15.8)$ \\
\hline Non-elective surgery, $N(\%)$ & $32(21.9)$ \\
\hline Number of comorbid conditions, mean (s.d.) & $1.8(1.0)$ \\
\hline Diabetes mellitus, $N(\%)$ & $25(17.1)$ \\
\hline Hepatic, $N(\%)$ & $10(6.8)$ \\
\hline Gastrointestinal, $N(\%)$ & $34(23.3)$ \\
\hline Renal, $N(\%)$ & $14(9.6)$ \\
\hline Respiratory, $N(\%)$ & $59(40.4)$ \\
\hline Cardiovascular, $N(\%)$ & $74(50.7)$ \\
\hline Neurological, $N(\%)$ & $39(26.7)$ \\
\hline NUTRIC score, mean (s.d.) & $4.1(1.7)$ \\
\hline LOS hospital, days, mean (s.d.) & $15.0(16.0)$ \\
\hline LOS ICU, days, mean (s.d.) & $29.6(26.8)$ \\
\hline Hospital mortality, yes, $N$ (\%) & $20(13.7)$ \\
\hline ICU mortality, yes, $N(\%)$ & $16(11.0)$ \\
\hline \multicolumn{2}{|l|}{ Patients with non-nutritional calories } \\
\hline Dextrose infusion ${ }^{\mathrm{a}}, N(\%)$ & $131(89.7)$ \\
\hline Propofol ${ }^{\mathrm{a}}, N(\%)$ & $101(69.2)$ \\
\hline Citrate dialyses ${ }^{\mathrm{a}}, N(\%)$ & $12(8.2)$ \\
\hline
\end{tabular}

Abbreviations: APACHE II, Acute Physiology and Chronic Health Evaluation; BMI, body mass index; ICU, intensive care unit; LOS, Length of Stay; NUTRIC, Nutrition Risk in Critically ill; SOFA, Sequential Organ Failure Assessment. Values are in mean (s.d.) and $N(\%)$. ${ }^{\mathrm{a}}$ Non-nutritional calories during the first 7 days of ICU admission.

The mean NUTRIC score was 4.1 (s.d. \pm 1.7$).{ }^{9}$ More characteristics are depicted in Table 2.

Nutritional and NNCs in all patients

All patients had low caloric intakes on the first day of ICU admission; the median value was $205 \mathrm{kcal}$ (IQR 48-443 kcal). On day 1 , the mean proportion of NNCs was $31.7 \%$. Nutritional caloric intake increased gradually to full support around day 3 (Figures 1 and 2). Mean highest caloric intakes from propofol and dextrose were observed on the second day $(81$ and $107 \mathrm{kcal}$, respectively), stabilising at a lower level around day 4. Mean caloric intake due to trisodium citrate gradually increased during the first week after ICU admission (Table 3).

Patients with renal replacement therapy (trisodium citrate CVVH) Total nutritional and NNC intake in the first week are depicted in Table 4a. Among patients with renal replacement therapy (trisodium citrate $\mathrm{CVVH}$; the citrate group, $n=12$ ), median total energy intake over 7 days because of trisodium citrate was $495 \mathrm{kcal}$ (IQR 245-1860 $\mathrm{kcal})$, reflecting a mean of $141 \mathrm{kcal}$ per day. Median caloric intake over 7 days from dextrose was $340 \mathrm{kcal}$ (IQR 148-675 kcal) and for propofol it was $55 \mathrm{kcal}$ (IQR 0-235 kcal). Comparing this subgroup with all patients who did not receive trisodium citrate $(N=135)$, there was a significant difference between the total NNCs $(1589 \mathrm{kcal}$ (s.d. \pm 792 ) vs $684 \mathrm{kcal}$ (s.d. \pm 595$), P<0.001$; Table 4b). The caloric intake from trisodium citrate gradually rose over time during the first week. At day 7, mean energy of trisodium citrate was $263 \mathrm{kcal}$ (s.d. \pm 208 ) (see Table 3); this is $17.8 \%$ of the total caloric intake on day 7. The proportion of NNCs on day 1 is $28.5 \%$ of the total intake and from day 3 this levelled off at 17-22\% (Figure 1).

Patients with dextrose infusion

Among patients with dextrose infusions $(N=131)$, median intake from dextrose over 7 days was $380 \mathrm{kcal}$ (IQR 200-550 kcal), from propofol it was $100 \mathrm{kcal}$ (IQR $0-360 \mathrm{kcal}$ ) and from trisodium citrate it was $0 \mathrm{kcal}$ (IQR $0-0 \mathrm{kcal}$ ). There were no significant differences in NNC intake between the dextrose infusion group and the group without dextrose infusion $(767 \mathrm{kcal}($ s.d. \pm 629$)$ vs $625 \mathrm{kcal}$ (s.d. \pm 858$), P=0.54$; Table 4 c). On day 1 , the proportion of NNCs was $48.6 \%$ (s.d. \pm 35.9 ), and this levelled off at about $6 \%$ around day 5 (Figure 1).

Patients with propofol infusion

Among patients with propofol $(N=100)$ administration, median intake from propofol during the first 7 days was 230 (IQR 85-595) kcal, from dextrose it was $380 \mathrm{kcal}$ (IQR 165-605 kcal) and from trisodium citrate it was $0 \mathrm{kcal}$ (IQR 0-0 kcal). Compared with the group without propofol, we observed significant differences between the NNCs $(915 \mathrm{kcal}$ (s.d. \pm 672$)$ vs $400 \mathrm{kcal}$ (s.d. \pm 446$)$, $P \leqslant 0.001)$, and also significant differences in the amount of dextrose infusions between the two groups (415 kcal (s.d. \pm 339$)$ vs $292 \mathrm{kcal}$ (s.d. \pm 211 ), $P=0.008$; Table 4d). During the first day, the proportion of NNCs was $49.4 \%$ (s.d. \pm 34.1 ), gradually decreasing to $6 \%$ on day 4 (Figure 1).

\section{Prediction model}

A total of 115 patients stayed in the ICU for at least 7 days. We excluded 31 patients (length of stay $<7$ days).

In univariate analysis, we found significant effects for the following baseline covariates associated with a total NNC intake of at least $5 \mathrm{kcal} / \mathrm{kg}$ during the first 7 days: age, days between hospital admission and ICU admission, sepsis, admission type (urgent surgery), MUST score and haemoglobin level (Table 5).

In the multivariate analysis, the only factor significantly predicting NNC intake during the first week was male gender and odds ratio of 0.31 (95\% Cl: 0.11-0.89). The energy from NNCs was higher in women: male vs female 0.91 (s.d. 7.49) vs 12.01 (s.d. 10.86), $P$-value 0.096 .

\section{DISCUSSION}

A total of 146 mechanically ventilated critically ill patients were included in the study. Of these, only 4 patients (2.7\%) did not receive any NNCs during the first 7 days after ICU admission, suggesting that NNC intake is common in the ICU.

The mean proportion of any three of the NNC sources on the first day of ICU admission was relatively large: $31.7 \%$. This can be explained by the fact that the proportion is mainly affected by low caloric feeding that is typically present during the first days after admission. While gradually increasing nutritional intake, the average NNC intake levelled off at $\sim 6 \%$ of the total calories on day 4. Although the proportion of NNC may be substantial early after ICU admission, the total caloric intake still is low, thus minimising the risk of overfeeding. However, when nutritional support is advancing to target the risk of overfeeding may increase when also NNCs are provided to ICU patients.

The 'propofol' group and 'dextrose' group are comparable to the total group with an NNC intake of $\sim 35 \%$ of the total amount 
Patients with citrate renal replacement therapy $(\mathrm{N}=12)$

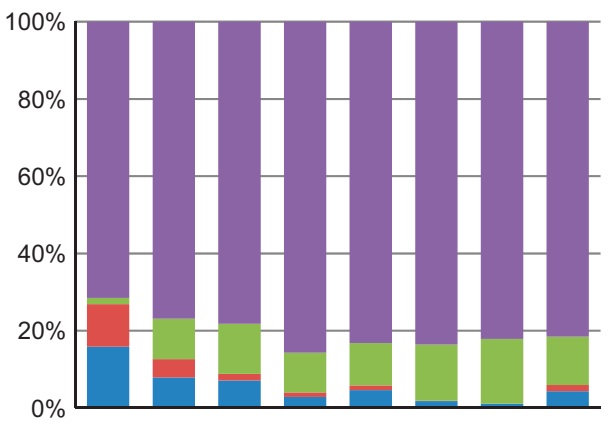

day 1 day 2 day 3 day 4 day 5 day 6 day 7 total

Patients with propofol $(\mathrm{N}=101)$

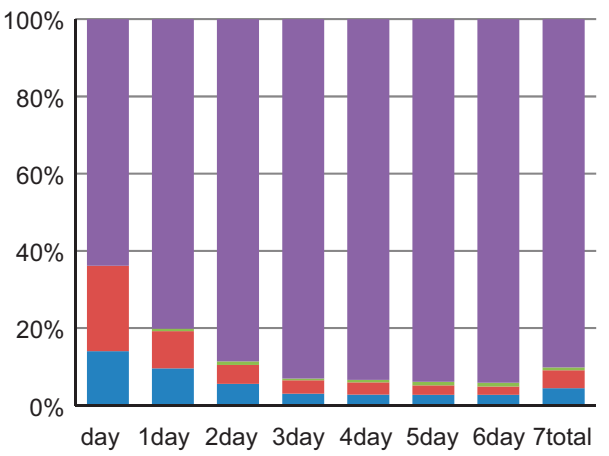

Patients with dextrose infusion $(\mathrm{N}=131)$

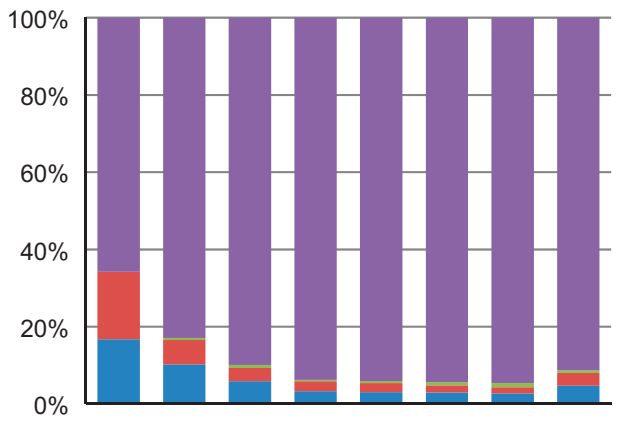

day 1 day 2 day 3 day 4 day 5 day 6 day 7 total

All Patients ( $=146)$

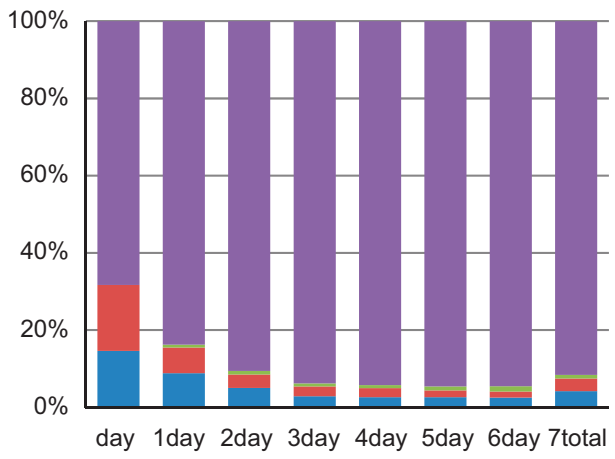

nutrition citrate propofol dextrose

Figure 1. Proportions of nutritional and NNCs in the first 7 days of ICU admission. On the $X$ axis, the day of ICU admission and the total of the first 7 days of admission, and on the $\mathrm{Y}$ axis, the calories from dextrose (blue), propofol (red), citrate (green) and nutrition (purple) of the total calories.

of calories on day 1 levelling off at $\sim 6 \%$ on days 4 and 5 . However, propofol administration and thus the contribution to NNCs is highly variable and is markedly associated with the actual sedation regimen of the institution.

However, in the 'citrate' group NNC intake at day 1 is slightly lower (28\%). However, this proportion is more stable during the first 7 days at $\sim 18 \%$ and even gradually increases over days. This may reflect the development of acute kidney injury and the need to start CRRT. Typically, in patients with acute kidney injury, oliguria or complications of renal insufficiency may be encountered over days after ICU admission. Therefore, citrate anticoagulation will commence later than, for example, propofol administration, a common sedative in mechanically ventilated patients. In addition, the administration of citrate will continue for days. We noticed a gradual increase in citrate administration over days, reflecting our protocol aiming at a level of hypocalcaemia in the filter to reach an anticoagulation level sufficiently to prevent clotting in the dialysis filter, leading to larger infusion rates of citrate over time.

When comparing those patients on dextrose infusionstypically $5 \%$ was used-with those without dextrose administrations, no statistically different caloric intake was noticed. Therefore, monitoring dextrose calories does not seem relevant, although when recommending to abandon monitoring dextrose calories it should be considered that no high-dose (10-50\%) dextrose administration was used in our patients.

NNC intake in critically ill patients cannot be easily predicted with an acceptable accuracy. On the basis of multivariate analysis, only female gender seemed to increase the odds of NNC intake during the first week after ICU admission.

Weijs et al. ${ }^{10}$ reported in a study among 843 intensive care patients (307 patients were overfed) a daily energy intake of $132 \mathrm{kcal}$ per day from non-nutritional sources (propofol and dextrose infusion). NNCs comprised $7.9 \%$ of total energy intake (6.4\% in overfed patients vs $10.1 \%$ in non-overfed patients). We found a lower average daily intake of $115 \mathrm{kcal}$ per day from non-nutritional sources. This is remarkable, as our data not only reflect the energy from propofol and dextrose infusion but from trisodium citrate as well, and therefore a higher intake would be expected. We found a proportion of $9.9 \%$ of the total energy intake. Notably, Ravasco and Camilo ${ }^{11}$ reported energy intake from NNCs (only propofol and dextrose infusion) to be $659 \mathrm{kcal}$ per day, a larger proportion of $32.4 \% .^{11}$ However, this was a relatively small study of 44 ICU patients. Taken together, these findings clearly suggest that large variability in NNCs may be encountered in individual patients, thus underlining the importance of individual patient monitoring for the most relevant sources of NNCs.

Our results should be valued in the context of the ongoing debate on optimal nutritional support for critically ill patients in the first week of ICU admission. ${ }^{12}$ Should we aim for full nutritional support or is trophic feeding acceptable ${ }^{13}$

In the EPaNIC trial, Casaer et al. ${ }^{14}$ compared 2312 adult critically ill patients with early initiation of parenteral nutrition with 2328 patients with late initiation to supplement enteral nutrition not reaching goals. They found that late initiation of supplemental parenteral nutrition was associated with faster recovery and fewer 


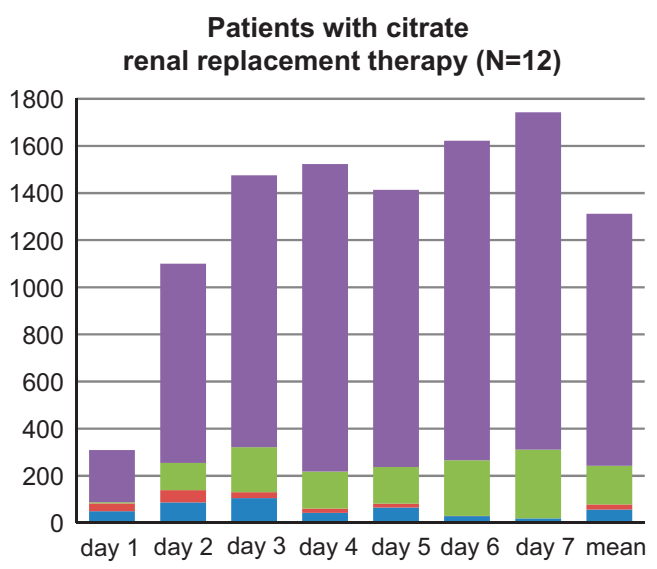

Patients with propofol $(\mathrm{N}=101)$

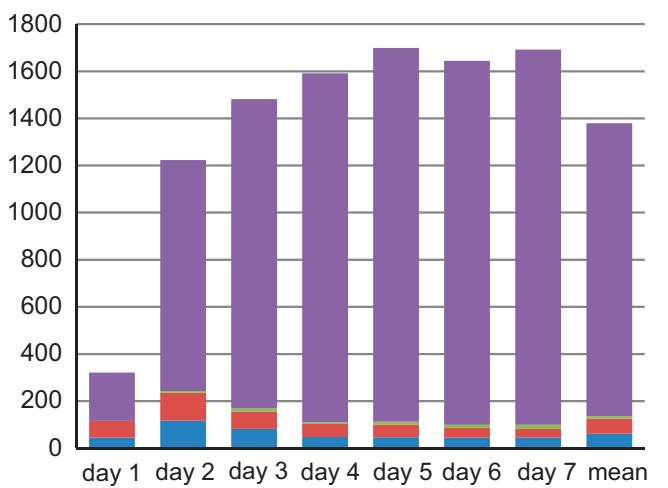

nutrition

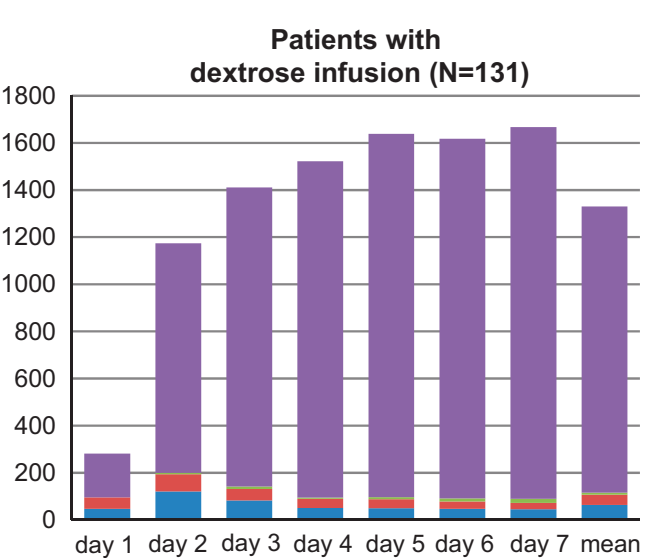

All Patients ( $\mathrm{N}=146)$

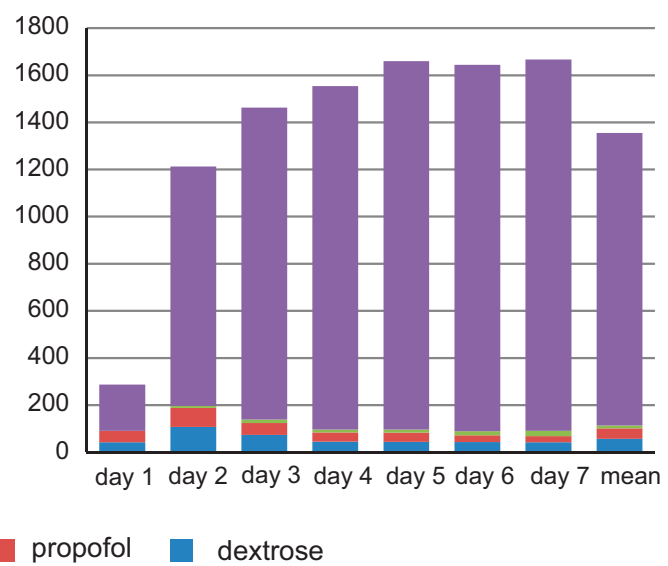

Figure 2. Absolute calories (nutritional and non-nutritional) of the first 7 days of ICU admission. On the $X$ axis, the day of ICU admission and the mean of the first 7 days, and on the $Y$ axis, the kcal per day from dextrose (blue), propofol (red), trisodium citrate (green) and nutrition (purple).

complications. Arabi et al. ${ }^{15}$ assigned 894 critically ill adult patients from seven centres to permissive underfeeding $(40-60 \%$ of the calculated target) or standard enteral feeding (70-100\% of the calculated target) for up to 14 days while maintaining the protein intake in both groups. They found that the 90-day mortality was similar- $27.2 \%$ in the permissive-underfeeding group and $28.9 \%$ in the standard-feeding group died (relative risk with permissive underfeeding, $0.94 ; 95 \% \mathrm{Cl}$ : 0.76-1.16; $P=0.58)-$ suggesting that trophic feeding is as good as full feeding. However, from observational data, it is known that increasing caloric intake and protein intake early after ICU admission only confers lower mortality in patients with BMls $<25$ and $>35 \mathrm{mg} / \mathrm{kg}^{2}{ }^{26}$ In all the studies, performed on trophic feeding, patients were slightly overweight (BMI $25-30 \mathrm{mg} / \mathrm{kg}^{2}$ ) and with low numbers of patients with high nutritional risk as expressed by high NUTRIC scores. ${ }^{13}$ This could imply that in these patients trophic feeding may be acceptable; however, this concept has not been proven in high-risk patients (lower and higher BMI classes and high NUTRIC scores). Therefore, as also no harm of full feeding except for early delivery through the parenteral route has been demonstrated, full nutritional support can still be recommended. ${ }^{13}$

Moreover, Singer et al. ${ }^{17}$ performed the TICACOS trial, comparing a study group receiving mainly enteral nutritional support based on energy targets determined by repeated indirect calorimetry measurements $(n=65)$ and a control group targeting $25 \mathrm{kcal} / \mathrm{kg}$ per day $(n=65)$. They found that patients in the indirect calorimetry group achieved higher mean energy (2086 vs
$1480 \mathrm{kcal}$ per day, $P=0.01$ ) and protein intake (76 vs $53 \mathrm{~g}$ per day, $P=0.01)$, associated with a trend toward improved hospital mortality $(32.3 \%$ vs $47.7 \%, P=0.058)$, whereas length of ventilation (16.1 vs 10.5 days, $P=0.03$ ), ICU stay ( 17.2 vs 11.7 days, $P=0.04$ ) and the amount of infectious complications (37 vs 20, $P=0.05$ ) were increased. More studies are ongoing.

As the jury is still out on optimal nutritional support in critical illness, and whether trophic or full nutrition can be recommended, there is general belief that hypercaloric feeding should be avoided, as this is associated with increased morbidity and mortality. Therefore, close monitoring of NNCs to prevent overfeeding is of pivotal importance.

\section{Strengths and limitations}

Although some research has been carried out on NNCs with respect to propofol and dextrose infusions, a strength of this study is the inclusion of calories from citrate administration. Tight monitoring of caloric intake from nutritional sources allowed a precise calculation of daily caloric intake from nutrition, allowing detailed calculations of both nutritional and NNCs. Therefore, we were able to exactly calculate proportions.

However our study has several limitations. Because of the retrospective observational design, some bias may have been introduced. We have tried to circumvent this by including consecutive patients meeting the inclusion criteria. Although we could not demonstrate any effect of the pre-post period of 
Table 3. Nutritional calories and the most relevant non-nutritional caloric sources ${ }^{a}$

\begin{tabular}{|c|c|c|c|c|c|c|c|c|c|c|c|c|c|c|}
\hline & \multicolumn{14}{|c|}{ Days of ICU admission } \\
\hline & \multicolumn{2}{|c|}{1} & \multicolumn{2}{|c|}{2} & \multicolumn{2}{|c|}{3} & \multicolumn{2}{|c|}{4} & \multicolumn{2}{|c|}{5} & \multicolumn{2}{|c|}{6} & \multicolumn{2}{|c|}{7} \\
\hline & Mean & (s.d.) & Mean & (s.d.) & Mean & (s.d.) & Mean & (s.d.) & Mean & (s.d.) & Mean & (s.d.) & Mean & (s.d.) \\
\hline \multicolumn{15}{|l|}{ All patients $(\mathrm{n}=146)$} \\
\hline Dextrose & 42 & (66) & 107 & (110) & 74 & $(83)$ & 45 & (68) & 44 & (73) & 43 & (77) & 42 & (71) \\
\hline Total NNCs & 91 & (120) & 197 & (197) & 139 & (155) & 96 & (135) & 97 & $(132)$ & 90 & (138) & 91 & (136) \\
\hline Nutrition & 196 & (239) & 1016 & (548) & 1325 & (625) & 1458 & (619) & 1564 & $(560)$ & 1556 & (543) & 1576 & (549) \\
\hline Total calories & 289 & (300) & 1213 & (552) & 1455 & (606) & 1554 & (628) & 1661 & (544) & 1643 & (528) & 1663 & $(560)$ \\
\hline \multicolumn{15}{|l|}{ Citrate $^{\mathrm{b}}(\mathrm{n}=12)$} \\
\hline Dextrose & 58 & (74) & 97 & (114) & 108 & (106) & 46 & (75) & 68 & (96) & 36 & (66) & 22 & (48) \\
\hline Propofol & 31 & (44) & 48 & (111) & 23 & (59) & 17 & (40) & 16 & (46) & 1 & (3) & 0 & (0) \\
\hline \multicolumn{15}{|l|}{$\operatorname{Dextrose}^{\mathrm{b}}(\mathrm{n}=131)$} \\
\hline Dextrose & 47 & (68) & 120 & (110) & 83 & (84) & 50 & (69) & 49 & (74) & 47 & (79) & 45 & (73) \\
\hline Propofol & 49 & (88) & 74 & (135) & 48 & (103) & 39 & (94) & 39 & (99) & 31 & (94) & 27 & (93) \\
\hline Trisodium citrate & 0 & $(0)$ & 6 & (41) & 11 & (59) & 6 & (46) & 9 & (60) & 13 & (72) & 17 & $(80)$ \\
\hline Total NNCs & 96 & (124) & 200 & (190) & 142 & (149) & 94 & (128) & 97 & $(131)$ & 91 & (138) & 89 & (132) \\
\hline Nutrition & 185 & (235) & 974 & (534) & 1269 & (613) & 1427 & (614) & 1541 & (559) & 1527 & $(542)$ & 1578 & (539) \\
\hline Total calories & 280 & (302) & 1174 & (539) & 1400 & (599) & 1521 & (620) & 1638 & (546) & 1615 & (527) & 1646 & (567) \\
\hline \multicolumn{15}{|l|}{ Propofol $^{b}(n=101)$} \\
\hline Dextrose & 45 & (67) & 117 & (120) & 81 & (86) & 47 & $(68)$ & 48 & (79) & 45 & $(81)$ & 47 & (76) \\
\hline Propofol & 71 & (96) & 117 & (167) & 72 & (128) & 55 & (108) & 53 & (109) & 39 & (103) & 35 & (103) \\
\hline Trisodium citrate & 0 & $(0)$ & 8 & (47) & 14 & (67) & 7 & (52) & 11 & (67) & 14 & (79) & 16 & $(80)$ \\
\hline Total NNCs & 115 & (132) & 241 & (212) & 167 & (167) & 109 & (138) & 112 & (140) & 99 & (148) & 98 & (139) \\
\hline
\end{tabular}

Abbreviations: ICU, intensive care unit; NNCs, non-nutritional calories. Values are in mean (s.d.). ${ }^{a}$ During the first 7 days of ICU admission, classified to

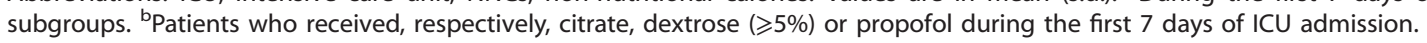

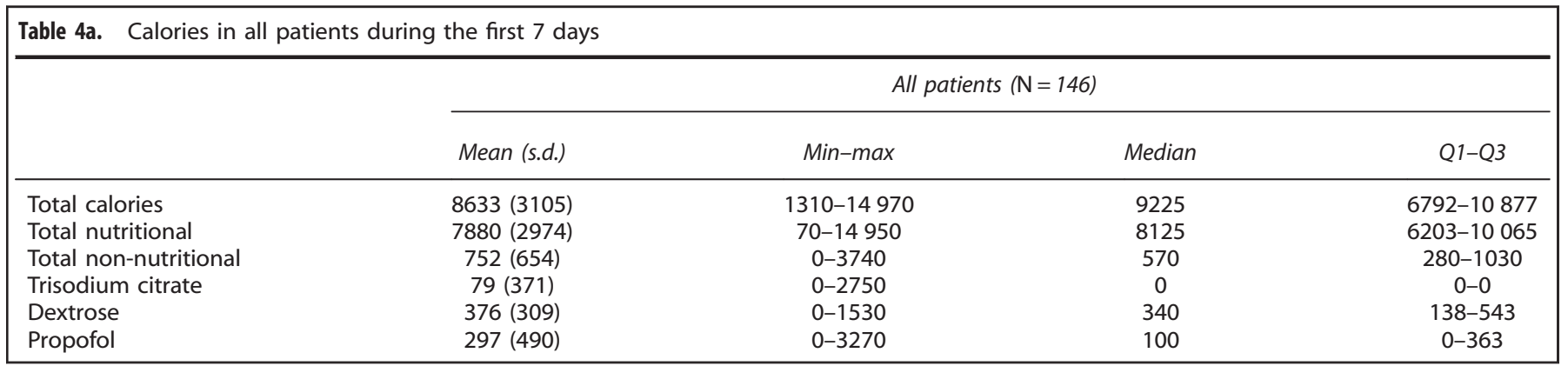

protocol implementation on NNC intake in our cohort, an effect in individual patients cannot be ruled out. Furthermore, patients with an ICU LOS of at least $72 \mathrm{~h}$ were only included, limiting generalisability for patients with shorter LOS. In addition, caloric targets of patients were not based on measurements of resting energy expenditure (REE) but by estimation using the WHO/FAO/ UNU equations. As energy expenditure formulae often overestimate the caloric needs of patients, this may only strengthen the role of NNCs, as when actual resting energy expenditure is lower the contribution of NNC is more relevant. Our selection criteria ultimately resulted in inclusion of only 12 patients who received CRRT. Therefore, the impact of trisodium citrate on NNCs needs more research, and present results preclude strong recommendations. Furthermore, CRRT itself may also affect energy expenditure, by decreasing energy expenditure through cooling of blood in the extracorporeal circuit conferring lower body temperatures. However, increases in energy expenditure may arise when patients counteract drops in body temperature in the presence of an extracorporeal circuit. Last, also nutrient loss across haemofilters has been described. 


\begin{tabular}{|c|c|c|c|c|c|c|c|c|c|c|c|c|c|c|c|c|c|c|}
\hline Total calories & 8557 & (3390) & 1770 & - & 11590 & 10125 & 5485 & - & 11010 & 8639 & (3095) & 1310 & - & 14970 & 9115 & 6793 & -10833 & 0.93 \\
\hline Total nutritional & 6986 & (3241) & 70 & - & 10410 & 7945 & 4365 & - & 9443 & 7953 & (2952) & 840 & - & 14950 & 8180 & 6203 & -10188 & 0.30 \\
\hline Total non-nutritional & 1589 & (792) & 610 & - & 3180 & 1490 & 792 & - & 2043 & 684 & (595) & 0 & - & 3740 & 530 & 260 & -933 & $<0.001$ \\
\hline Propofol & 146 & (195) & 0 & - & 570 & 55 & 0 & - & 235 & 309 & (505) & 0 & - & 3270 & 100 & 0 & 370 & 0.29 \\
\hline Total (excluding citrate) & 535 & (447) & 0 & - & 1320 & 580 & 165 & - & 828 & 684 & (595) & 0 & - & 3740 & 530 & 260 & 933 & 0.42 \\
\hline
\end{tabular}

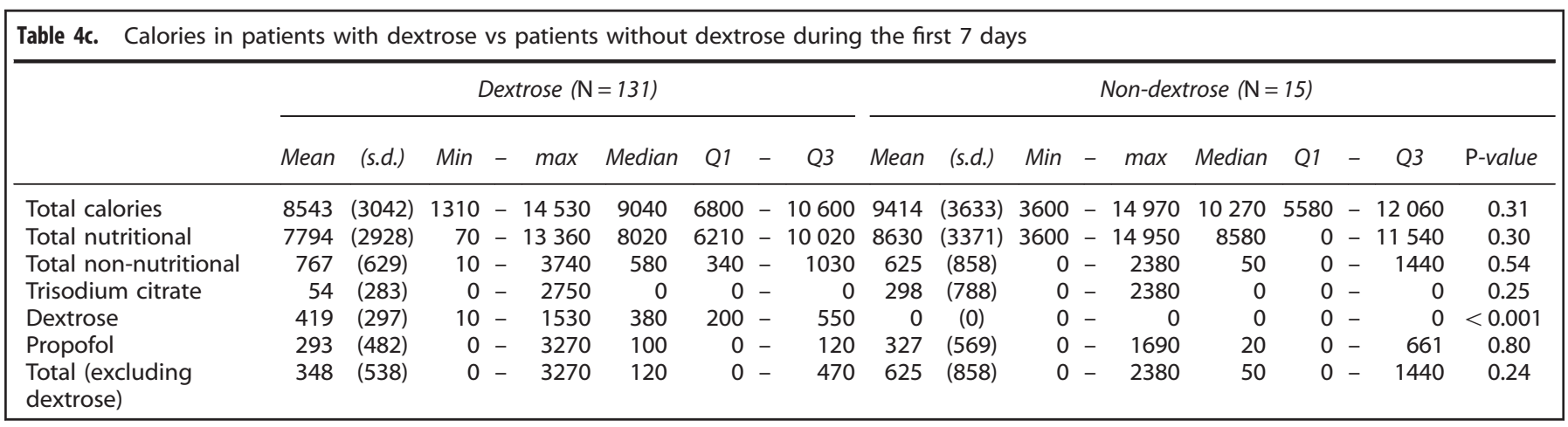

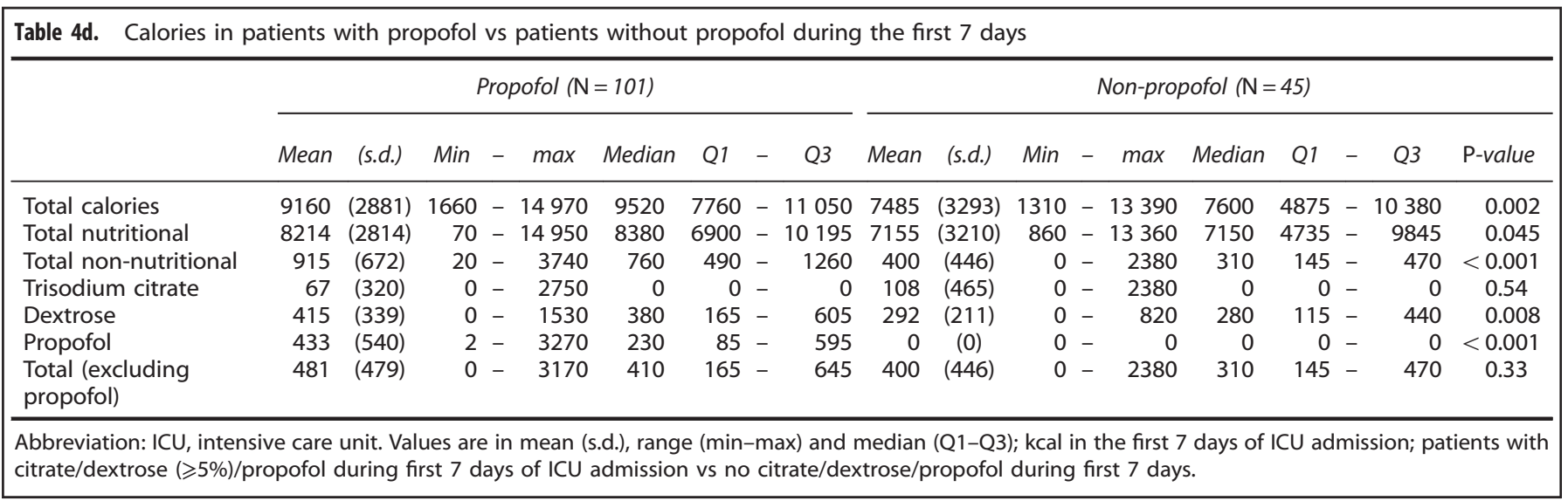

\begin{tabular}{|lccl|}
\hline Table 5. Multivariate prediction analysis of baseline characteristics & ${ }^{\mathrm{a}}$ \\
\hline Covariates & Odds ratio & $95 \% \mathrm{Cl}$ & P-value \\
\hline Gender (male) & 0.31 & $0.11-0.89$ & 0.029 \\
Age at admission (year) & 1.03 & $0.99-1.07$ & 0.12 \\
Pre-ICU hospitalisation (days) & 1.10 & $0.96-1.26$ & 0.15 \\
Admission type (surgical vs medical) & 0.59 & $0.32-1.07$ & 0.081 \\
MUST score (per point) & 1.01 & $0.71-1.43$ & 0.968 \\
Haemoglobin (mmol/l) & 0.91 & $0.64-1.29$ & 0.604 \\
\hline
\end{tabular}

Abbreviations: $\mathrm{Cl}$, confidence interval; ICU, intensive care unit; MUST, Malnutrition Universal Screening Tool. Prediction analysis for more than 5 kcal energy from NNCs per $\mathrm{kg}$ during the first 7 days of ICU admission; ${ }^{1}$ Days between hospital admission and ICU admission. ${ }^{\mathrm{a}}$ For energy from non-nutritional calories during the first week after ICU admission.

\section{CONCLUSIONS}

NNC intake on day 1 after ICU admission is large; however, because of low caloric feeding while advancing nutritional support, the overfeeding risk is low. The mean proportion of NNCs in patients who receive dextrose and/or propofol is low (6\%) during the first week; however, in individual patients it may comprise one-third of the total daily calories. Monitoring dextrose calories seems irrelevant. Patients receiving trisodium citrate for renal replacement therapy anticoagulation have higher mean non-nutritional intakes ( 18\%). Predicting higher non-nutritional intake on ICU admission is difficult if not possible at all, although women in our study seem to have higher NNC intake. As this intake can be marked in individual patients, close monitoring is warranted when administering high-dose propofol or trisodium 
citrate anticoagulation to prevent overfeeding, particularly when nutritional support is reaching set energy targets.

\section{CONFLICT OF INTEREST}

$E B, D v B$ and HJWL declare no conflict of interest. Dr van Zanten reported that he has received honoraria for advisory board meetings, lectures and travel expenses from Abbott, Baxter, Braun, Danone-Nutricia, Fresenius Kabi and Nestle-Novartis. Inclusion fees for patients in nutrition trials were paid to the local ICU research foundation.

\section{AUTHOR CONTRIBUTIONS}

Dr van Zanten had full access to all of the data in the study and takes responsibility for the integrity of the data and the accuracy of the data analysis. Study concept and designing: EB, ARHvZ. Acquisition of data: EB, ARHvZ and HJWL. Statistical analysis and interpretation of data: EB and ARHvZ. Drafting the manuscript: EB and ARHvZ. Critical revision of the manuscript for important intellectual content: $E B$ and ARHvZ. Administrative, technical or material support: EB and DvB. Study supervision: EB and ARHvZ.

\section{REFERENCES}

1 Barr J, Hecht M, Flavin KE, Khorana A, Gould MK. Outcomes in critically ill patients before and after the implementation of an evidence-based nutritional management protocol. Chest 2004; 125: 1446-1457.

2 McClave SA, Taylor BE, Martindale RG, Warren MM, Johnson DR, Braunschweig C et al. Guidelines for the Provision and Assessment of Nutrition Support Therapy in the Adult Critically III Patient: Society of Critical Care Medicine (SCCM) and American Society for Parenteral and Enteral Nutrition (A.S.P.E.N.). J Parenter Enteral Nutr 2016; 40: 159-211.

3 McClave SA, Weijs PJ. Preservation of autophagy should not direct nutritional therapy. Curr Opin Clin Nutr Metab Care 2015; 18: 155-161.

4 Food and Agriculture Organization of the United Nations (2003). Food energymethods of analysis and conversion factors-Annex IV. Available at: ftp://ftp.fao. org/docrep/fao/006/y5022e/y5022e00.pdf (last accessed 30 September 2015).

5 Greenwood J. ICU Guideline: adjusting goal feed rates in the patients receiving propofol, 2009. Available at: http://www.criticalcarenutrition.com/docs/tools/Pro pofol.pdf (last accessed 28 September 2015).

6 Letteri JL, Huang Z, Ronco C, Gao D, Clark W. Solute and water kinetics in continuous therapies. In: Ronco C, Bellomo R, Kellum JA (eds). Critical Care Nephrology. 2nd edn Elsevier, Saunders: 2009, pp 1377-1385.

7 Keefe Davis T, Neumayr T, Geile K, Doctor A, Hmeil P. Citrate anticoagulation during continuous renal replacement therapy in pediatric critical care. Pediatr Crit Care Med 2014; 15: 471-485.
8 Bousie E, van Blokland D, van Zanten ARH. Effects of implementation of a computerized nutritional protocol in mechanically ventilated critically ill patients: a single-centre before and after study. Clin Nutr ESPEN 2016; e47-e54.

9 Heyland DK, Dhaliwal R, Jiang X, Day AG. Identifying critically ill patients who benefit the most from nutrition therapy: the development and initial validation of a novel risk assessment tool. Crit Care 2011; 15: R268.

10 Weijs PJM, Looijaard WG, Beihuizen A, Girbes AR, Oudemans-van Straaten HM. Early high protein intake is associated with low mortality and energy overfeeding with high mortality in non-septic mechanically ventilated critically ill patients. Crit Care 2014; 18: 701.

11 Ravasco P, Camilo ME.. The impact of fluid therapy on nutrient delivery: a prospective evaluation of practice in respiratory intensive care. Clin Nutr 2003; 22: 87-92.

12 Preiser JC, van Zanten AR, Berger MM, Biolo G, Casaer MP, Doig GS et al. Metabolic and nutritional support of critically ill patients: consensus and controversies. Crit Care 2015; 19: 35.

13 Van Zanten AR. Full or hypocaloric nutritional support for the critically ill patient: is less really more? J Thorac Dis 2015; 7: 1086-1091.

14 Casaer MP, Mesotten D, Hermans G, Wouters PJ, Schetz M, Meyfroidt G et al. Early versus late parenteral nutrition in critically ill adults. $N$ Engl J Med 2011; 365: 506-517.

15 Arabi YM, Aldawood AS, Haddad SH, Al-Dorzi HM, Tamim HM, Jones G et al. Permissive Underfeeding or Standard Enteral Feeding in Critically III Adults. N Engl J Med 2015; 372: 2398-2408.

16 Alberda C, Gramlich L, Jones N, Jeejeebhoy K, Day AG, Dhaliwal R et al. The relationship between nutritional intake and clinical outcomes in critically ill patients: results of an international multicentre observational study. Intens Care Med 2009; 35: 1728-1737.

17 Singer P, Anbar R, Cohen J, Shapiro H, Shalita-Chesner M, Lev S et al. The Tight Calorie Control Study (TICACOS): a prospective, randomized controlled pilot study of nutritional support in critically ill patients. Intens Care Med 2011; 37: 601-609.

(i)(2) This work is licensed under a Creative Commons AttributionConCommercial-ShareAlike 4.0 International License. The images or other third party material in this article are included in the article's Creative Commons license, unless indicated otherwise in the credit line; if the material is not included under the Creative Commons license, users will need to obtain permission from the license holder to reproduce the material. To view a copy of this license, visit http:// creativecommons.org/licenses/by-nc-sa/4.0/

(c) The Author(s) 2016 